\title{
Simulative verification of a novel semi-active broadband energy harvester
}

\author{
K. Retan ${ }^{1}$, A. Graf ${ }^{1}$, and L. Reindl ${ }^{2}$ \\ ${ }^{1}$ ZF Friedrichshafen AG Graf-von-Soden-Platz 188046 Friedrichshafen, Germany \\ ${ }^{2}$ IMTEK Lehrstuhl für Elektrische Mess- und Prüfverfahren Georges-Köhler-Allee 106, 79110 Freiburg, \\ Germany
}

Correspondence to: A. Graf (alexander.graf@zf.com)

Received: 18 September 2013 - Revised: 13 December 2013 - Accepted: 16 December 2013 - Published: 17 January 2014

\begin{abstract}
This paper presents a semi-active broadband vibrational-energy harvesting system. Based on a nonresonant rotational generator, electronic circuitry was used to overcome the physical start-up restrictions. Due to the functional design it remains an energy harvester suitable for battery-less devices. For the first time a vibrational energy harvester is presented that allows standardization and thus higher volume production. A system layout, simulation, and measurement data will be shown.
\end{abstract}

1

Wireless sensor systems have become an essential component in the field of structural health monitoring (SHM). These devices offer continuous information regarding the mechanical stability of structures ranging from automobiles to large buildings. Using a wireless transceiver, data can be gathered and used to evaluate structural integrity, which allows for accurate predictions of the structure's life expectancy and facilitates the timely repair of damaged components (Yang et al., 2007; mon, 2013).

Due to the limited lifespan of battery powered systems, energy harvesting systems have been developed, which transform environmental energy into electrical power. The classic implementation of this idea is the solar cell, which generates an electric potential from solar radiation. This energy can then be used to power system components directly or recharge a battery, which can dramatically improve the system's life expectancy. In addition to solar power, thermal gradients and vibrational forces can be exploited. The optimal energy solution depends on the application environment.

Where sunlight or thermal gradients are sporadic or nonexistent, vibrational energy can be used to generate power needed for wireless systems. Ambient vibrations are present in a variety of environments, ranging from structures such as bridges and buildings to industrial machines and house- hold appliances. This form of energy harvesting generally uses the mechanical energy from a vibrating body to excite a spring-mass system, which then oscillates with the frequency of the vibration source. The systems exhibit high power output at vibration frequencies very close to the harvester's resonant frequency but are very inefficient outside this small frequency range. Examples of commercial resonant energy harvesters include the PMG Free Standing Harvester from Perpetuum (Per, 2012), as well as the Volture from MIDE (Stephen, 2006).

The application environment, however, can exhibit a wide range of vibration frequencies, necessitating a factory tuning of the harvester to match the device's resonant frequency with that of the environment. Additionally, the dominant vibration frequency is not necessarily static, which disqualifies these devices from use in environments in which the vibration frequency does not remain within a very narrow bandwidth.

To increase the operating bandwidth of kinetic energy harvesters, recent efforts have focused on incorporating nonlinear effects into the harvester system. Xing et al. (2011) recently published results from a cantilever harvester constructed of a high-permeability magnetic material (Xing et al., 2011). The cantilever was mounted horizontally inside a solenoid. Two permanent magnets were placed near the tip such that tip oscillations would cause a periodic 


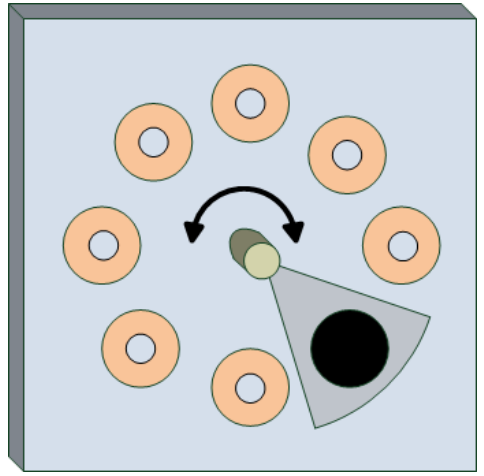

Figure 1. Pendulum-based non-resonant energy harvester developed by Spreemann et al. (2009).

magnetization reversal in the cantilever. The combination of magnetostatic and elastic effects increased the bandwidth of the system to $10 \mathrm{~Hz}$ while maintaining a peak power density of $1.07 \mathrm{~mW} \mathrm{~cm}^{-3}$.

Additionally, fully non-resonant harvesting concepts have been investigated. Bowers and Arnold (2009) constructed a rolling-magnet generator, which consisted of a spherical magnet enclosed in a spherical cavity, which was then wrapped in copper wiring. The device was designed to generate energy from human motion, which is characteristically aperiodic and varies greatly over time. A voltage of up to $700 \mathrm{mV}$ was generated, which equated to a power density of up to $0.5 \mathrm{~mW} \mathrm{~cm}^{-3}$.

Finally, Spreemann et al. (2009) developed a kind of pendulum harvester (cp. Fig. 1) which operated using complete rotations rather than small-amplitude oscillations. In terms of broadband characteristics, this harvester was very successful, exhibiting an operating frequency range from 30 to $80 \mathrm{~Hz}$ at output powers between 0.4 and $3 \mathrm{~mW}$. A significant design flaw, namely the inability to establish this rotational state automatically, prevented the generator from becoming a viable energy harvesting solution.

Therefore, this paper focuses on the start-up functionality and describes the corresponding system layouts.

\section{Basic pendulum theory}

To understand the broadband characteristics of pendulumbased energy harvesters a basic knowledge of their dynamic behavior is required. The equation of motion for a damped mathematical pendulum excited by vibrational forces is given by (Gitterman, 2010):

$\ddot{\varphi}+c \dot{\varphi}+\frac{g}{l} \sin \varphi=F \cos (\omega t)$,

where $\varphi, \dot{\varphi}$ and $\ddot{\varphi}$ denote the angular position, velocity and acceleration, respectively. The constants $c$ and $l$ represent the damping coefficient and length of the pendulum, respectively, while $g$ stands for the gravitational constant. The term

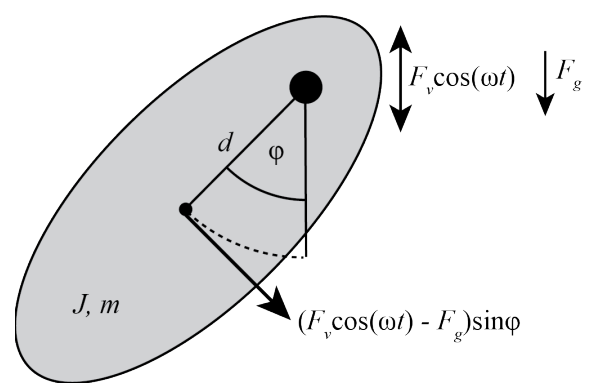

Figure 2. Physical pendulum described by its mass $(\mathrm{m})$, moment of inertia $(J)$ and center of mass $(d)$.

$F \cos (\omega t)$ represents the force driving the pendulum, which is assumed to be sinusoidal with amplitude $F$ and angular frequency $\omega$. This equation describes a pendulum which consists of a point mass at the end of a massless pendulum arm and is driven by a force tangential to pendulum's path.

In order to describe a physical pendulum driven by a vibrational force (see Fig. 2), several modifications were made to this equation. First, the pendulum was described as an object with nonzero dimensions using its moment of inertia. Second, the vibrational force is restricted to a purely vertical motion, which means it is no longer tangential to the path of rotation. The coupling of the driving force into the pendulum system is a function of the pendulum's current position and is described by the prefactor $\sin (\varphi)$. Finally, the sum of all driving or damping moments originating in the transducer is represented by $M_{\mathrm{T}}$. These moments will be investigated in detail in Sect. 2.2. After making these modifications, the resulting equation of motion can be written as

$\ddot{\varphi} J+M_{\mathrm{T}}+m g d \sin \varphi=-A \omega^{2} m d \cos (\omega t) \sin (\varphi)$,

with the constants $m, J$, and $d$ representing the pendulum's mass, moment of inertia and the distance of the center of mass from the pivot, respectively. $A$ and $\omega$ characterize the amplitude and the frequency of the vibration.

Given a pendulum which is initially motionless and modest vibration intensity, the pendulum oscillates around the resting position. For small oscillations, the angular position is close to zero, and thus prefactor $\sin (\varphi)$, which determines the coupling of the vibration forces into the pendulum system, is small. For energy harvesting, this is an inherently inefficient generation state, as very little mechanical energy is transferred into the system. As the amplitude of the vibrations increases, so does the amplitude of the pendulum oscillations, and thus also the coupling factor as it approaches the maximum value of one.

In order to maximize the efficiency of the pendulum as an energy harvester, the coupling of the mechanical energy of a given vibration into the pendulum system must be maximized. This is realized by matching the phase of the pendulum's oscillations with that of the vibrations, which implies 


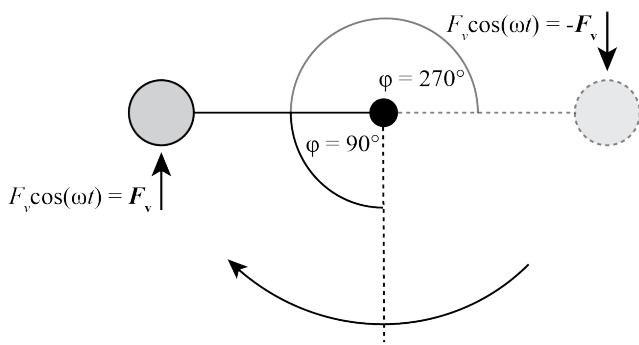

Figure 3. Values of $\varphi$ for which the transfer of vibrational energy is maximized.

$\varphi=\omega t+\frac{\pi}{2}$

Physically, the phases of the vibrations and the pendulum are matched when the pendulum performs complete rotations with a frequency equal to that of the vibrations and a phase shift of $\pi / 2$. When the vertical vibration force reaches its maximum amplitude at $\omega t=0$, the pendulum is completely horizontal, enabling maximum transfer of vibrational moment into the system as depicted in Fig. 3.

At this point, the transfer of energy is efficient enough that the vibrations can sustain the pendulum's rotation for certain levels of damping. As Spreemann et al. (2009) discovered, energy transfer in this state is so efficient that rotation can be maintained while simultaneously extracting meaningful amounts of energy from the system in the form of electricity. This rotational state is therefore the preferred state for pendulum-based energy harvesting, as it maximizes energy transfer into the system to the point that surplus energy can be extracted with an appropriate transduction mechanism.

The pendulum's equation of motion, like most nonlinear differential equations, does not possess a closed solution (Gitterman, 2010), and the classical linearization assuming small deflection angles is not valid in this case. Therefore, numerical simulations of the pendulum's behavior were conducted to investigate its suitability as an energy harvesting solution. Simulations of the pendulum's rotational behavior will be covered in Sect. 2.1.

\subsection{System concept}

The proposed energy harvester concept requires a level of system complexity which exceeds that of traditional resonant energy harvesters. The components required for the proposed semi-active harvester are shown in a block diagram in Fig. 4. As stated previously, in order for the harvester to efficiently generate energy its angular velocity must be matched to the dominant vibration frequency. The system must therefore possess the ability to both accelerate the pendulum and generate power. The simplest and most elegant solution available, which was also selected for the proposed energy harvesting system, consists of a DC-motor with an attached pen-

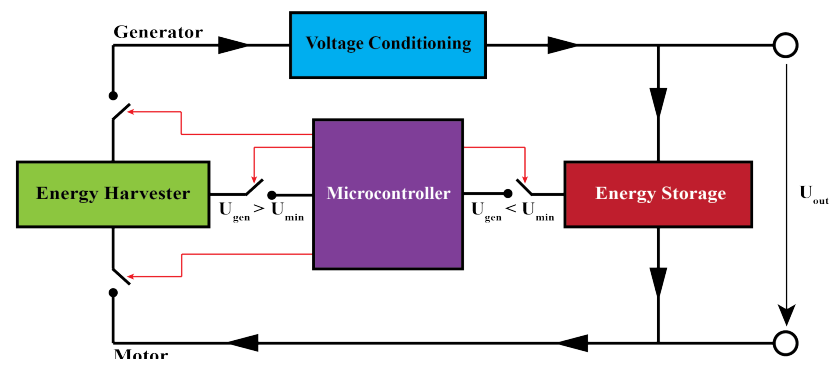

Figure 4. Block diagram of the system layout.

dulum. By controlling the direction of energy flow to and from the motor, it can be used initially to accelerate the pendulum and subsequently as the transduction mechanism for energy harvesting.

Additionally, control electronics and a suitable algorithm are necessary to regulate the harvester's rotational speed, to ensure its rotational stability and to control energy flow to and from the energy storage. Generated voltages must be conditioned for energy storage, necessitating the use of a voltage converter. Control electronics can be powered either directly from the harvester or from the energy storage, depending on the harvester's output voltage, which is a function of its operating frequency.

\subsection{Modeling of components}

\subsubsection{DC motor}

A simplified model was used for the DC motor. Inductive effects were intentionally omitted due to the motor's small electrical time constant, which is given by

$\tau_{\mathrm{e}}=\frac{L_{\text {motor }}}{R_{\text {motor }}}$

with $L_{\text {motor }}$ and $R_{\text {motor }}$ representing the motor's inductance and internal resistance, respectively. The DC motors considered for the energy harvester typically have a resistance in the order of $100 \Omega$ and induction of several hundred $\mu \mathrm{H}$, yielding time constants of less than $5 \mu \mathrm{s}$. This is very small compared to the vibration frequencies expected in the target application, which should not exceed $100 \mathrm{~Hz}$. Inductive effects would therefore have a negligible influence on the motor's behavior and are not necessary for modeling. Motor rotation induces a voltage $V_{\mathrm{EMF}}$, which is proportional to the rotational velocity according to

$V_{\mathrm{EMF}}=k_{\mathrm{E}} \dot{\varphi} \frac{60}{2 \pi}$

where $V_{\text {motor }}, \dot{\varphi}$ and $k_{\mathrm{E}}$ denote the motor voltage, motor velocity in radians per second and the back EMF constant in $\mathrm{V} \mathrm{rpm}^{-1}$, respectively. The constant $k_{\mathrm{E}}$ is an experimentally determined, intrinsic property of every DC motor and is listed in its datasheet. In motor operation this voltage opposes 
the applied voltage, effectively increasing the internal resistance of the motor. In generator operation this is the measured output voltage at a given rotational velocity. The motor current is then given by

$I_{\text {motor }}=\frac{V_{\text {op }}-V_{\mathrm{EMF}}}{R_{\text {motor }}+R_{\text {load }}}=\frac{V_{\text {op }}-k_{\mathrm{E}} \dot{\varphi}}{R_{\text {motor }}+R_{\text {load }}} \cdot \frac{60}{2 \pi}$,

where $I_{\text {motor }}, V_{\text {op }}, R_{\text {motor }}$ and $R_{\text {load }}$ represent the motor's current, operating voltage, internal resistance and load resistance, respectively. During motor operation the load resistance can be ignored, which simplifies Eq. (6) to

$I_{\text {motor }}=\frac{V_{\mathrm{op}}-V_{\mathrm{EMF}}}{R_{\text {motor }}}$.

During generator operation the operating voltage is zero, which reduces Eq. (6) to

$$
I_{\text {motor }}=\frac{V_{\mathrm{EMF}}}{R_{\text {motor }}+R_{\text {load }}} \text {. }
$$

A second constant $k_{\mathrm{I}}$, which is termed the current constant and is given in units $\left(\mathrm{A} \mathrm{mN} \mathrm{m}^{-1}\right)$, relates this current flow to motor torque. As with the back-EMF, $k_{\mathrm{I}}$ is specific to each motor and is listed in its datasheet. Using Eq. (6) and constant $k_{\mathrm{I}}$, the current-induced motor moment can be expressed as

$M_{\mathrm{E}}=I_{\text {motor }} \cdot \frac{1}{k_{\mathrm{I}}}=\frac{V_{\mathrm{op}}-k_{\mathrm{E}} \dot{\varphi}}{R_{\text {motor }}+R_{\text {load }}} \cdot \frac{60}{2 \pi} \cdot \frac{1}{k_{\mathrm{I}}}$,

where $M_{\mathrm{E}}$ denotes the electrical contribution to the motor moment. When the DC motor is operated as such during the energy harvester's acceleration phase, this moment causes rotational acceleration. When the motor is operated at a generator, this moment opposes the vibration-induced rotation and thus can be seen as a viscous damping force, as it is proportional to the motor's rotational speed. In addition to the electrical moment $\left(M_{\mathrm{E}}\right)$, a mechanical damping $\left(M_{\mathrm{M}}\right)$ was included to model the friction in the motor bearings. This friction moment is largely independent of rotational speed and thus is included as a constant moment $\left(c_{\mathrm{m}}\right)$ which always opposes the direction of rotation.

$M_{\mathrm{M}}=c_{\mathrm{m}} \cdot \operatorname{sgn}(-\dot{\varphi})$

The sum of the electrical and mechanical moments in the DC motor,

$M_{\mathrm{T}}=M_{\mathrm{E}}+M_{\mathrm{M}}$,

can then be inserted into Eq. (2), yielding the complete pendulum energy harvester equation of motion which was used in the simulation model.

\subsubsection{Voltage conditioning}

A boost converter was necessary to increase the generator voltage to the point that it could be stored in a lithium ion

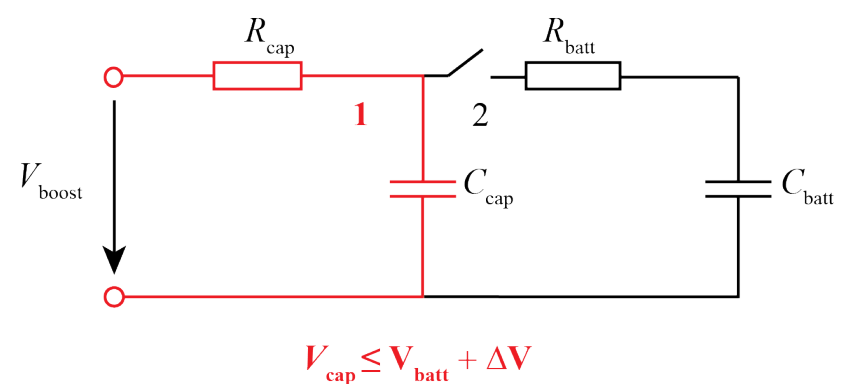

Figure 5. Initial state of the battery charging circuit.

battery. Boost converters are circuits which utilize switching transistors and inductors to increase voltage. These circuits typically operate at $\mathrm{MHz}$ frequencies, and thus their dynamic behavior has little impact on the relatively low-frequency energy harvester system. For the sake of simplicity, a boost converter was modeled which generated a fixed output voltage $\left(V_{\text {out }}\right)$ and exhibited a certain efficiency $(\eta)$, which is defined as

$\eta=\frac{P_{\text {out }}}{P_{\text {in }}}$.

In reality, the efficiency on the input voltage, decreasing as it approaches the minimum input voltage, below which the converter ceases to function. For modeling purposes, an average efficiency was estimated for the harvester's output voltage range using efficiency values from component datasheets.

\subsubsection{Energy storage and charging circuit}

The energy storage and charging circuit simulation blocks were implemented using the differential equation for capacitor charging, which is given by

$V_{\mathrm{c}}(t)=i(t) R+\frac{1}{C} \int_{0}^{t} i(\tau) \mathrm{d} \tau$,

where $V_{\mathrm{c}}, i(t), R$ and $C$ denote the capacitor voltage, capacitor current series resistance and capacitance, respectively. Capacitance and resistance can be approximated using an exponential fit of the charging characteristic found in the battery's datasheet.

The charging circuit was designed to have a minimum number of active components while still effectively charging the battery. In the proposed energy harvesting system, the output voltage of the boost converter is used to charge the battery. For the simulation model, an output voltage of $5 \mathrm{~V}$ was selected. To charge the thin-film battery, the charging voltage must exceed the current battery voltage $\left(V_{\text {batt }}\right)$ while not exceeding the maximum voltage rating $\left(V_{\max }\right)$ of the battery in order to avoid damaging the battery. 


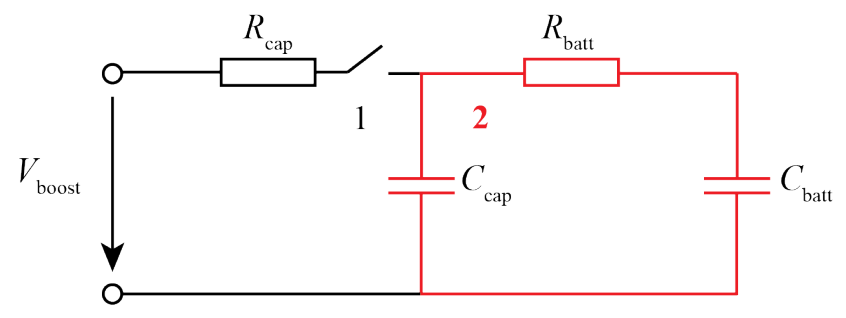

$$
V_{\text {cap }} \geq V_{\text {batt }}
$$

Figure 6. Transition state of the battery charging circuit.
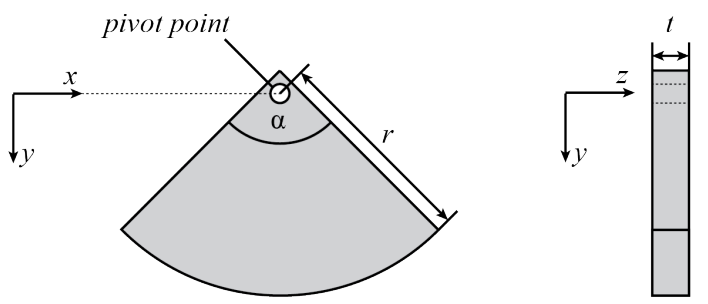

Figure 7. Geometry and orientation of the rotational mass.

To this end, the charging circuit depicted in Figs. 5 and 6 was used. It consists of the energy storage component with charging voltage $V_{\text {batt }}=4.1 \mathrm{~V}$, a capacitor and two switches. Initially, switch 1 is closed and switch 2 is open. The capacitor is thus charged to a value $V_{\text {batt }}+\Delta V$ above the charging voltage and below the maximum rating. The switch states are then reversed, which results in the capacitor transferring its charge to the battery until its voltage is equal to or below that of the battery voltage $V_{\text {batt }}$.

\subsection{Model verification}

The primary goal of preliminary simulations was the verification of the simulation model's accuracy. To this end, a simple pendulum generator was constructed using a cylindrical segment pendulum (cp. Fig. 7) and a Faulhaber DC motor.

By varying the opening angle $(\alpha)$, the radius $(r)$ and the thickness $(t)$ of the cylindrical segment, pendulums with various moments of inertia and centers of mass could be designed. The pendulum was designed to maximize vibration torque according to

$M_{\mathrm{vib}}=F_{\mathrm{vib}} d \sin \varphi$,

where $F_{\text {vib }}, d$, and $\varphi$ represent the instantaneous vibration force, the pendulum's center of mass and its angular position respectively. The center of mass $(d)$ and the total mass $(m)$ were thus chosen as large as possible without exceeding the motor bearing's tolerance of radial forces as described by:

$F_{r, \max }=m d \dot{\varphi}^{2}$,

where $\dot{\varphi}$ and $m$ denotes the mass and angular velocity of the pendulum. For simulation verification a 1016012 G DC

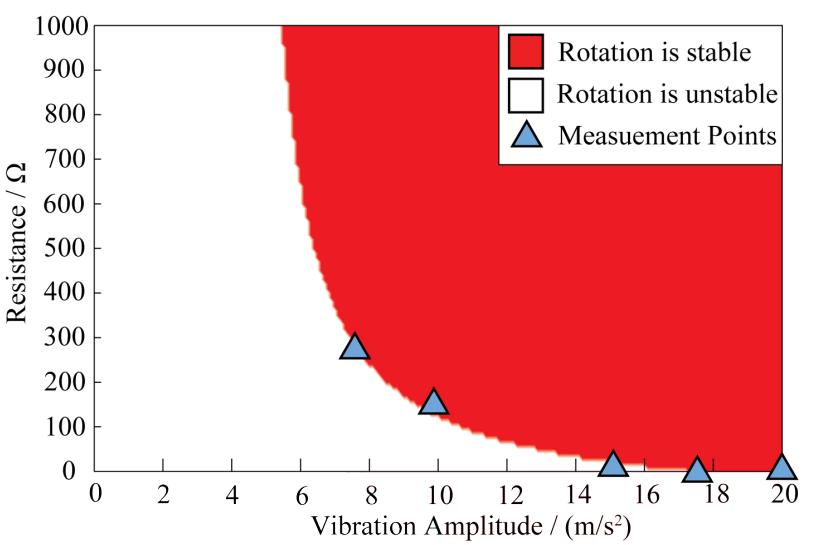

Figure 8. Verification of the simulation model.

motor from Faulhaber was selected due to its high radialload tolerance of $5 \mathrm{~N}$ (fau, 2012). The pendulum was designed such that its radial force would not exceed $5 \mathrm{~N}$ at the chosen maximum operating frequency of $100 \mathrm{~Hz}$. Based on this design criterion a steel pendulum with the dimensions $r=10 \mathrm{~mm}, \alpha=120^{\circ}$ and $t=3 \mathrm{~mm}$ was constructed, yielding a maximum radial force of $4.93 \mathrm{~N}$ at $100 \mathrm{~Hz}$. Using an electromagnetic shaker as a vibration source, the maximum current output of the generator was investigated at an operating frequency of $20 \mathrm{~Hz}$ and for amplitudes between 0.75 and $2 \mathrm{~g}$.

The pendulum was accelerated into a stable rotation using a finger flick. During the acceleration phase the generator load was $1 \mathrm{M} \Omega$, resulting in minimal electrical damping which facilitated phase matching. Once rotations were stable, the load was reduced from $1 \mathrm{k} \Omega$ in $10 \Omega$ steps until the pendulum fell out of rotation due to excessive electrical damping. This load was deemed the minimum tolerable electrical load for a given vibration frequency and amplitude (cp. Fig. 8).

Subsequently, the dimensions of the constructed pendulum as well as the damping characteristics of the selected DC motor, which are given in the corresponding datasheet (fau, 2012), were incorporated into the simulation model. Simulations were then conducted for which the pendulum was given an initial angular velocity and phase which were matched to the vibration frequency. As with the generator measurements, the vibration frequency was set to $20 \mathrm{~Hz}$, while the amplitude was varied between 0 and $2 \mathrm{~g}$. The electrical load was also varied between 0 and $1 \mathrm{k} \Omega$. A simulation time length of $10 \mathrm{~s}$ was chosen after it had been observed that this interval was adequate to determine whether or not the pendulum's rotation was truly stable at the given electrical load. Upon cessation of the simulation, the pendulum's angular velocity was investigated to determine whether or not the pendulum was still rotating.

Simulation results as well as the measurement results are shown in Fig. 8. The plot regions which are marked in red indicate stable rotation, whereas the white regions indicate a cessation of rotation. The red-white transition indicated the 


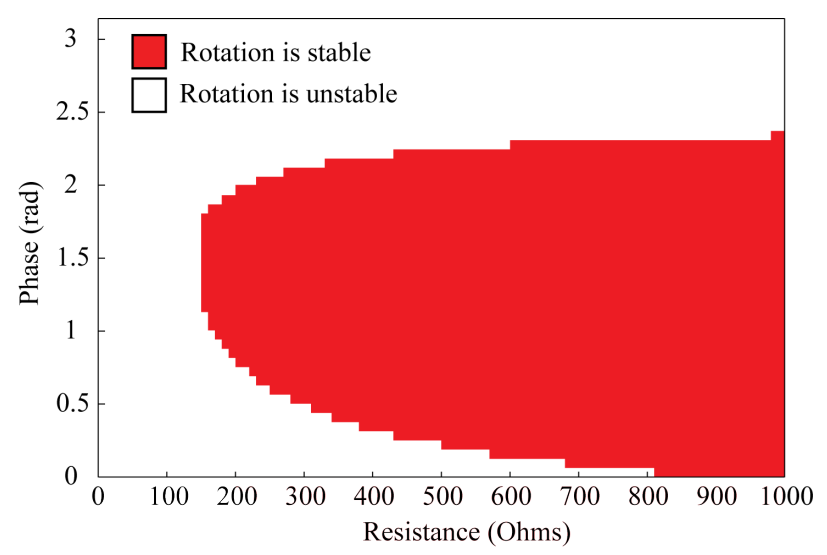

Figure 9. Investigation of the phase matching requirement as a function of the load resistance.

minimum electrical load for which rotations could be maintained for a given vibration amplitude. The good agreement between the simulation and measurement results was interpreted as a verification of the accuracy of the simulation model, which could therefore be used to further investigate the dynamics of the pendulum generator.

\section{Simulation and measurement results}

In this section the simulation model will be used to investigate different operational phases of the generator system. Based on these data, hardware was developed and tested.

\subsection{Simulation of the start-up phase}

After verifying the accuracy of the simulation model, additional system components necessary for generator start-up and energy storage can be included. As stated previously, the proposed energy harvester concept requires a substantial energy investment in order to accelerate the pendulum to its operating frequency. Thus an energy storage component is necessary to provide the initial energy investment and subsequently store generated energy. For this simulation model, the energy storage component was modeled after an MEC225 Thinergy thin-film lithium-ion battery from Infinite Power Solutions (Inf, 2012). Thin-film batteries generally exhibit much lower leakage currents than capacitors with comparable storage capacities, which increases the maximum tolerable system down-time between periods of vibration activity.

Additionally, a control strategy was necessary to match the motor speed to the vibration frequency. Initial simulations indicated that phase matching tolerances between the motor and vibrations become increasingly stringent as electrical loads decrease, which affect input energy and dissipated power, respectively (cp. Fig. 9). A reduction in vibration amplitude was also observed to decrease the tolerances for phase

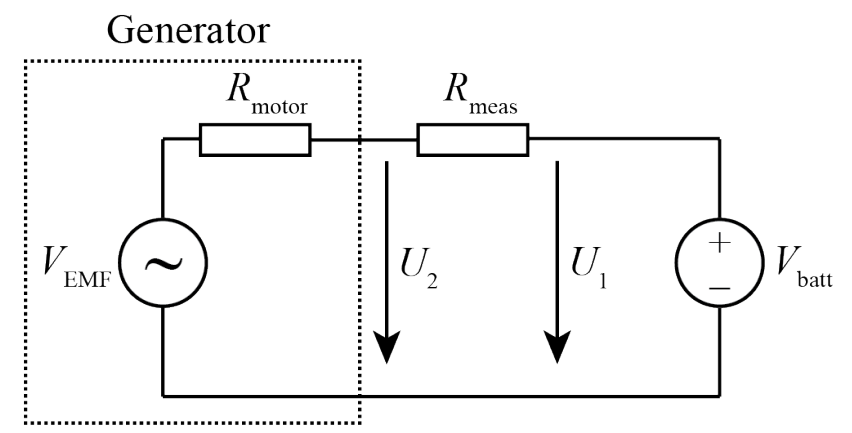

Figure 10. Equivalent circuit for the determination of $V_{\mathrm{EMF}}$

matching. Therefore, a very accurate control strategy could potentially allow for motor operation at lower vibration amplitudes. However, the benefits obtained from an accurate but complex control strategy must be weighed against the potential increases in the operation current. For a preliminary analysis, a two-point control strategy was used, which could be implemented on a very low current budget.

Therefore, the back EMF was calculated from voltage measurements first:

$V_{\mathrm{EMF}}=U_{1}-\frac{U_{1}-U_{2}}{R_{\text {meas }}} \cdot\left(R_{\text {motor }}+R_{\text {meas }}\right)$,

where $R_{\text {meas }}, R_{\text {motor }}, U_{1}$ and $U_{2}$ represent the current measurement resistor, internal motor resistance and two potential measurements shown in Fig. 10, respectively.

From the back EMF and the back EMF constant found in the motor's datasheet, the motor speed can be calculated according to

$f_{\text {motor }}=\frac{V_{\mathrm{EMF}}}{60 \cdot 2 \pi}$.

The control strategy itself functions as shown in Fig. 11. The vibration frequency $\left(f_{\text {target }}\right)$ represents the target average motor velocity, with the instantaneous motor velocity oscillating periodically around this value. Upper and lower velocity tolerances were then defined, which allow for the natural oscillatory variation around $f_{\text {target }}$. Starting from a standstill, the motor is first accelerated to the upper motor speed tolerance, at which point it is switched off. The velocity then decelerates through the tolerance range. If phase matching occurs during this deceleration, the motor remains within the velocity tolerances. Should the motor velocity decrease beyond the lower velocity tolerance, it is accelerated again and allowed to decelerate through the tolerance range again. This process is repeated until the motor achieves phase matching with the vibrations.

Once the control strategy was incorporated into the simulation model, simulations of the motor start-up phase were conducted to confirm the control strategy's effectiveness. Simulations were conducted at vibration frequencies of 20 , 40 and $60 \mathrm{~Hz}$ for a vibration amplitude of $1 \mathrm{~g}$. Results are 


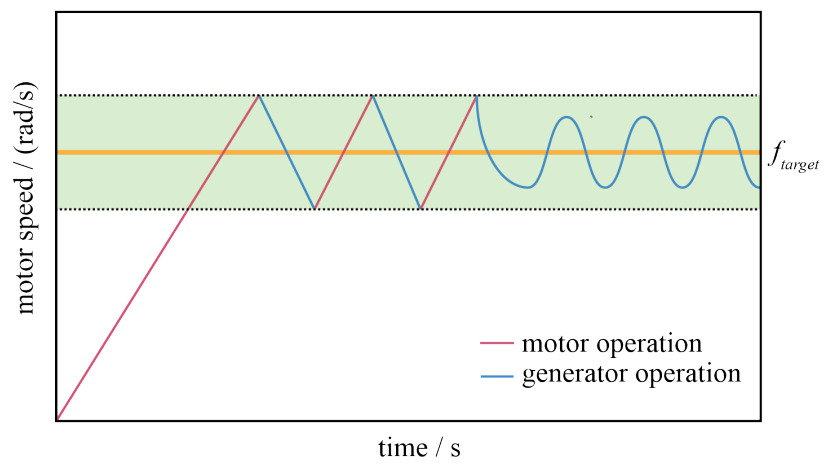

Figure 11. Desired transient response of the accelerated pendulum.

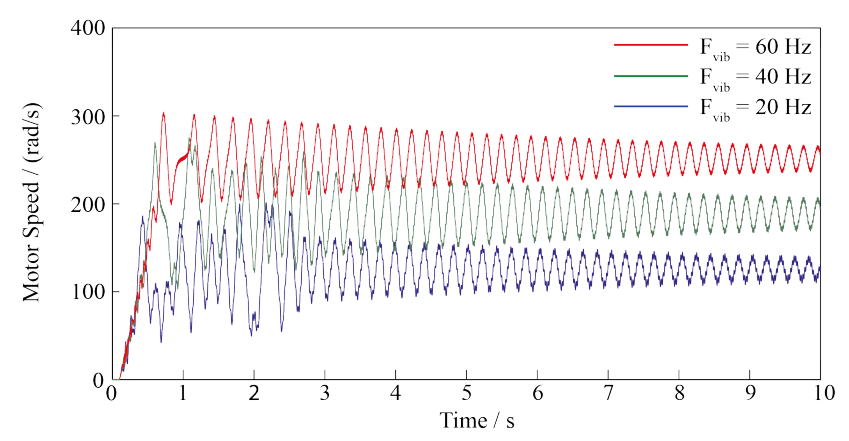

Figure 12. Simulated motor speed with respect to frequency and time.

shown in Figs. 12 and 13. The diagram in Fig. 12 shows the velocity of the motor over time, which increases initially to the target frequency and subsequently enters a stable rotation, as indicated by the stable average velocity. The diagram in Fig. 13 shows the balance of charge following through the motor.

A positive slope indicates current flowing to the motor, whereas a negative slope indicates that the motor is generating current that is then flowing to the energy storage. This plot can be used to determine how much energy is invested to accelerate the pendulum as well as how long the generator must run to recuperate the energy investment. These charge balance plots show that for a vibration amplitude of $1 \mathrm{~g}$, phase matching occurs relatively quickly. The current flowing to the energy storage, which is indicated by the slope of the plot, is $0.8,1.15$ and $1.3 \mathrm{~mA}$ for $f_{\mathrm{vib}}=20,40$ and $60 \mathrm{~Hz}$, respectively. The varying slopes of the charge balance curves arise from the fact that the generator's output voltage is proportional to its rotation frequency. Extrapolating the charge balance curves, the charge invested to start the generator can be recuperated in 20,10.7 and 5.4 seconds for the respective rotation frequencies.

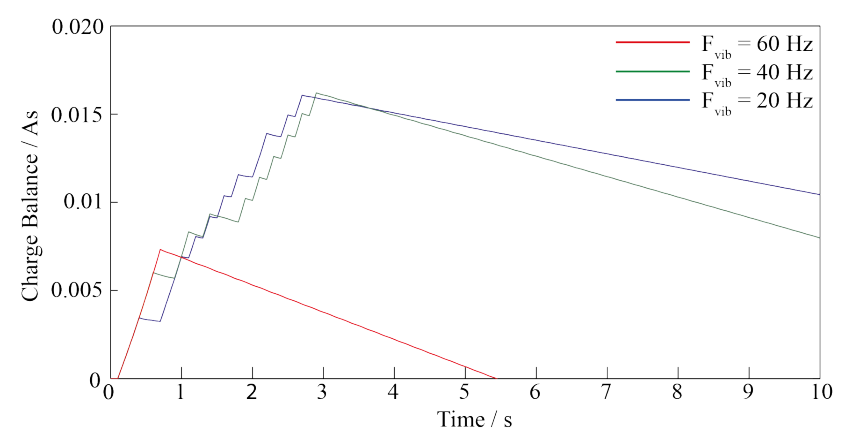

Figure 13. Simulated charge balance of the start-up phase.

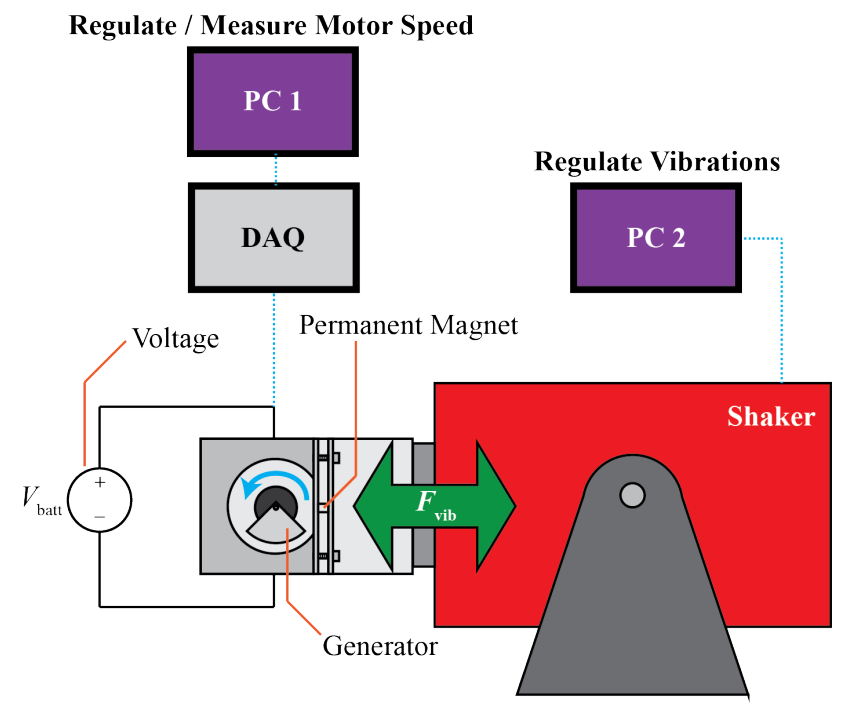

Figure 14. Setup of the measurement equipment.

\subsection{Measurements}

After verifying the control strategy's effectiveness using simulation results, the strategy was then implemented using a NI USB-6259 BNC data acquisition device (DAQ) from National Instrument, which was connected to a computer PC1. The control software was programmed in C. A voltage source set to $4.1 \mathrm{~V}$ was used to emulate the MEC225 battery. A $1 \mathrm{k}$ resistor was then used as an electrical load, which approximated the battery's resistive load. As a vibration source, an electromagnetic shaker was again used, which was controlled by a second computer PC2. The complete measurement setup can be seen in Fig. 14.

To facilitate the comparison with the simulation results, measurements were conducted a 20,40 and $60 \mathrm{~Hz}$ for a vibration amplitude of $1 \mathrm{~g}$. Measurement results are shown in Figs. 15 and 16.

As evident from the results, phase matching was achieved for all three frequencies. Compared to the simulations, the energy expended for acceleration was recuperated much more quickly. This is due to the fact that a simple load 


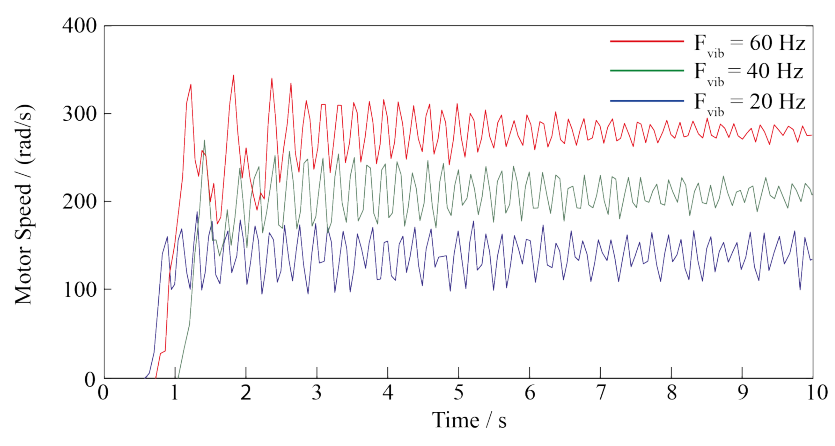

Figure 15. Measured motor speed with respect to frequency and time.

Table 1. Generator output power over Frequency.

\begin{tabular}{lllll}
\hline Frequency $(\mathrm{Hz})$ & 20 & 30 & 40 & 50 \\
\hline Power $(\mathrm{mW})$ & 16.9 & 10.6 & 5.8 & 6.8 \\
\hline
\end{tabular}

resistance with no capacitive behavior was used, allowing for much larger motor currents. Additionally, no boost converter was necessary for voltage preparation, which tends to reduce the output current in order to raise the output voltage. Despite these differences, these measurements experimentally demonstrate the effectiveness of the simple two-point control strategy for motor speed regulation.

Finally, the motor's maximum power output was investigated at various frequencies (cp. Table 1). The maximum power was defined as the minimum resistive load for which the generator could remain in rotation at a given vibration frequency and amplitude. The maximum output power of $16.9 \mathrm{~mW}$ was measured for $20 \mathrm{~Hz}$. The reduced power output at frequencies above $20 \mathrm{~Hz}$ is likely due to the measured frequency dependence of the friction in the motor bearings.

\section{Conclusions}

The simulation and measurement results presented in Sect. 3 confirm the functionality and broadband characteristics of pendulum-based energy harvesters. In addition, the proposed mechanism for accelerating the pendulum to its operating frequency was also demonstrated to be viable. Using the proposed control strategy, phase matching between the pendulum rotation and vibration frequency was achieved over a frequency range from $20-50 \mathrm{~Hz}$, allowing subsequent energy generation at power outputs greater than $5 \mathrm{~mW}$. This output power level is more than adequate for state-of-the-art wireless sensor systems. In conclusion, the semi-active pendulum-based energy harvester was proved to be a viable broadband vibration energy harvesting solution. Future research will be focused on the development of an autonomous pendulum harvester, which will possess the

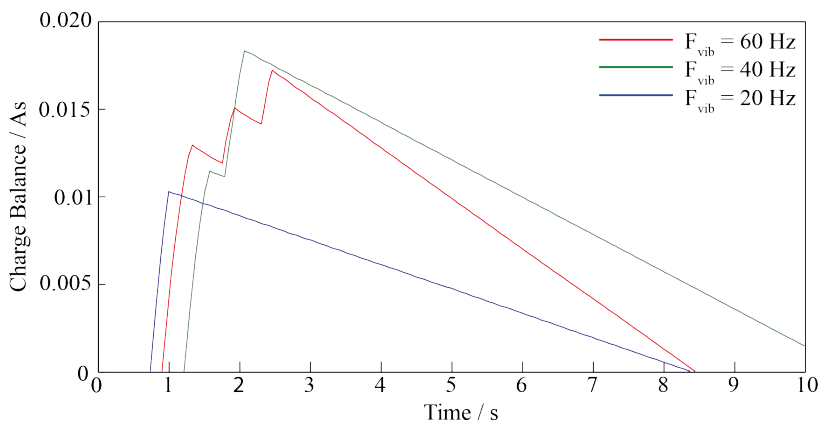

Figure 16. Measured charge balance of the start-up phase.

ability to autonomously detect ambient vibrations on an extremely low current budget and subsequently begin energy harvesting at the dominant vibration frequency.

Edited by: U. Schmid

Reviewed by: two anonymous referees

\section{References}

Bowers, B. J. and Arnold, D. P.: Spherical, rolling magnet generators for passive energy harvesting from human motion, J. Micromech. Microeng., 19, 1-7, 2009.

Serie 1016 DC-Kleinstmotoren mit Edelmetallkommutierung, Faulhaber, www.faulhaber.com, 2012.

Gitterman, M.: The Chaotic Pendulum, World Scientific Publishing Co. Pte. Ltd., 5 Toh Tuck Link, Singapore 596224, 2010.

MEC225 Solid-State, Flexible, Rechargeable ThinFilm Micro-Energy Cell, Infinite Power Solutions, www.infinitepowersolutions.com, 2012.

Wireless Sensor Power Management, http://www.monnit.com/blog, 2013.

PMG FSH Free Standing Harvester, www.perpetuum.com, 2012.

Spreemann, D., Manoli, Y., Folkmer, B., and Mintenbeck, D.: Nonresonant Vibration Conversion, Non-resonant Vibration Conversion, 16, 169-173, 2009.

Stephen, N.: On energy harvesting from ambient vibration, Journal of Sound and Vibration, 293, 409-425, 2006.

Xing, X., Yang, G. M., Liu, M., Lou, J., Obi, O., and Sun, N. X.: High power density vibration energy harvester with high permeability magnetic material, J. Appl. Phys., 109, 109-111, 2011.

Yang, W., Lynch, J., and Law, K.: A Wireless Structural Health Monitoring System with Multithreaded Sensing Devices: Design and Validation, Structure and Infrastructure Engineering: Maintenance, Management, Life-Cycle Design and Performance, 3, 103-120, 2007. 